\title{
Le Jeu des simulacres ... ou la rhetorique consciente : du Bellay-Ronsard
}

\author{
JEAN-CLAUDE MOISAN
}

Pelletier, dans son Art poëtique, écrit que "la matière de l'ode sont les louangés des Dieux, Démidieux, et des Princés : Les amours, les banquez, les jeuz festiz, et samblablés passétans. Qui montrét qu'elé et capablé de diuers argumants et de diuers stile. Car en parlant des Dieus et des Heroïns, ele se hausse : mes non pas jusqués au stile Heroïque. Car il i a diferancé dé diré les louangés des grans, et dé chanter leurs gestés. ..."1 Quelques lignes plus loin il situera l'ode "au déssous de l'euvre Heroïque"' avant de proclamer au début du chapitre sur l'Euvre Héroïque qu'il est "celui qui donne le prix, et le vrai titre de Poëte."

Il y a donc une hiérarchie en poésie et plus l'oeuvre que l'on crée s'approchera de la matière épique, plus elle sera considérée comme élevée.

C'est le cas des Hymnes de Ronsard, dont la plupart racontent les gestes de dieux et de héros et dont certains ont été considérés par Laumonier comme "de petites épopées" voire comme des "essais et des préludes de la Franciade" et dont les autres portent sur des sujets hautement philosophiques.

Par contre, dans les Regrets, du Bellay renonce "à entrer si avant en si profonds secretz" et se contentera, dit-il, "de simplement écrire."

"Simplement écrire," pour du Bellay, veut dire opposer sa pratique littéraire dans les Regrets (le sonnet 4 précise bien: en ce livre et la nuance est de taille) à celle des poètes inspirés, dont le Ronsard des Hymnes. C'est ainsi, que dès le début des Regrets, il opposera l'un à l'autre les termes suivants : ${ }^{6}$

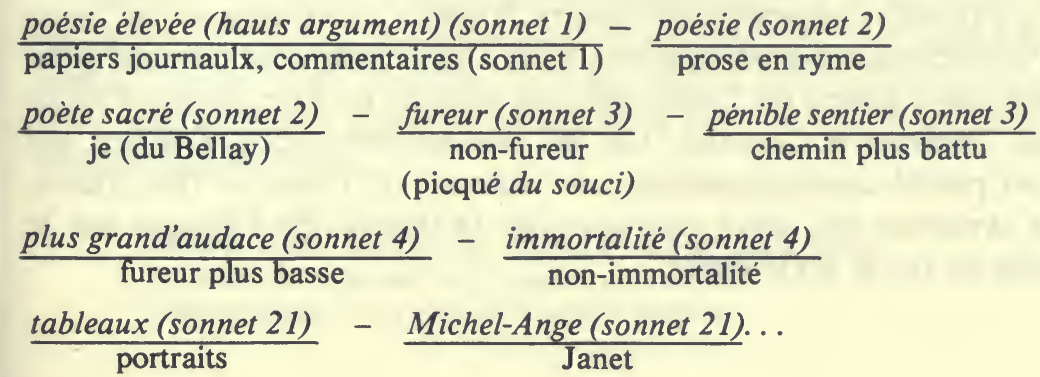


La dernière antithèse Michel-Ange/Janet renvoie à un autre texte de du Bellay fort éclairant, celui du Discours au Roy sur la Poésie :
Cestuy-là (l'historien) sans user d'aucune
fiction
Représente le vray de chacune action
Comme un, qui sans oser s'esgayer davantage
Rapporte après le vif un naturel visage :
Cestuy-cy plus hardi, d'un art non limité
Sous mille fictions cache la vérité ...
Tel que ce premier là est votre Janet, SIRE
Et tel que le second Michelange on peut dire

\section{Donc :}

$\frac{\text { poésie }}{\text { histoire }}-\frac{\text { Michel-Ange }}{\text { Janet }}-\frac{\text { tableau }}{\text { portrait }}-\frac{\text { poésie menteuse ou élevée }}{\text { papiers journaux }}$

Ces oppositions sont d'ailleurs reprises par Ronsard dans l'Epistre au lecteur de la Franciade :

J'ose seulement dire (si mon opinion a quelque poix) que le Poète qui escrit les choses comme elles sont ne mérite tant que celuy qui les feint et se recule le plus qu'il luy est possible de l'historien. ...8

Voilà pourquoi, comme du Bellay, mais pour des raisons inverses, il surmotivera son oeuvre, pour employer l'expression heureuse de Ménager, pour bien indiquer qu'il aspire, lui, à une poésie hors du commun.

Contrairement à du Bellay toutefois, il proclamera qu'il est "Tourmenté d'Apollon," "plein de fureur" qu'il va suivre "les pas d'Orphée" (Hymne de l'Eternité), ou encore, selon la rhétorique de l'époque, il demandera l'aide des Muses (Henry II) ; il enverra sa "Muse extravaguer par une estroitte voye," il affirmera que "l'argument est fort haut" (les Daimons). Lorsque le chant de païen devient chrétien, c'est le Dieu chrétien qui insuffle la flamme sacrée au poète (Hercule chrestien).

On le voit donc, les deux oeuvres (dans le cas des Hymnes, il s'agit bien sûr de presque chacun d'entre eux) commencent par un énoncé rhétorique et un " $\mathrm{je}$ " qui théorise sa pratique littéraire. Vouloir confondre ce " $\mathrm{je}$ " avec le sujet d'énonciation serait hasardeux. Il s'agit bien évidemment d'un simulacre au sens précis où l'entendent les auteurs du Dictionnaire de la sémiotique, Greimas et Courtés. ${ }^{9}$ Le "je" des Regrets et des Hymnes, qui théorise, est plutôt analogiquement un "acteur" de l'énoncé rhétorique, fruit d'une synthèse des idées courantes de la théorie de l'époque sur le style humble et sur le style élevé. 
On l'aura remarqué, il existe toutefois une première différence apparente entre les deux énoncés. Celui des Hymnes ne donne qu'un aspect de la dichotomie style humble/style élevé, alors que dans les Regrets se retrouvent les deux aspects de l'énoncé. C'est que dans cette oeuvre, le "je" acteur de l'énoncé rhétorique est pour ainsi dire double : celui du maintenant et de l'ici comparé à un "je" acteur d'un énoncé rhétorique antérieur. Il faut relire, dans cet optique, les passages suivants des sonnets six et sept pour s'en convaincre :

Las où est maintenant ce mespris de Fortune?

Où est ce coeur vainqueur de toute adversité

Cest honneste desir de l'immortalité,

Et ceste honneste flamme au peuple non commune?

Où sont ces doulx plaisirs, qu'au soir soubs la nuict brune

Les Muses me donnoient, alors qu'en liberté

Dessus le verd tapy d'un rivage esquarté

Je les menois danser aux rayons de la Lune?

Maintenant la Fortune est maistresse de moy,

Et mon coeur qui souloit estre maistre de soy,

Est serf de mille maulx \& regrets qui m'ennuyent.

De la posterité je n'ay plus de souci,

Ceste divine ardeur, je ne l'ay plus aussi,

Et les Muses de moy, comme estranges, s'enfuyent.

(Sonnet 6)

Ce pendant que la court mes ouvrages lisoit,

Et que la soeur du Roy, l'unique Marguerite,

Me faisant plus d'honneur que n'estoit mon merite,

De son bel oeil divin mes vers favorisoit,

Une fureur d'esprit au ciel me conduisoit

D'une aile qui la mort \& les siècles evite,

Et le docte troppeau qui sur Parnasse habite,

De son feu plus divin mon ardeur attisoit.

Ores je suis muet, comme on voit le Prophete

$\mathrm{Ne}$ sentant plus le Dieu, qui la tenoit sujette,

Perdre soudainement la fureur $\&$ la voix ....

(Sonnet 7)

Ce "je," "acteur" d'un énoncé rhétorique autre, réapparaîtra vers la fin des Regrets. Marguerite retrouvée, recommencent à poindre les notions de vertu, d'inspiration poétique et d'ascension vers les cieux. Ce "je" peut donc annoncer son nouveau projet situé hors du maintenant et de l'ici du recueil des Regrets :

Ains je veulx, comme toy, poingt d'un plus hault souci,

Chanter de ce grand Roy, dont le grave souci

Fait trembler le celeste, \& l'infernal empire. 
Je veulx chanter de Dieu. Mais pour bien le chanter,

Il fault d'un avant-jeu ses louanges tenter,

Loüant, non la beauté de ceste masse ronde,

Mais ceste fleur, qui tient encor' un plus beau lieu :

Car comme elle est (Du-val) moins parfaitte que Dieu,

Aussi l'est elle plus que le reste du monde.

Le "je" rhétorique des Hymnes par contre est un : il a toujours été un inspiré, il a toujours suivi la voie étroite, il était donc l'élu tout choisi pour chanter les Hymnes. Il faut lire l'Hymne de l'automne en ayant en tête ce que l'on vient de dire des Regrets pour comprendre. Donnons quelques exemples :

Le jour que je fu né, Apollon qui preside

Aux Muses, me servit en ce monde de guide,

M'anima d'un esprit subtil et vigoureux,

Et me fist de science et d'honneur amoureux.

Me donna pour partage une fureur d'esprit,

Et l'art de bien coucher ma verve par escrit.

Il me haussa le coeur, haussa la fantaisie,

M'inspirant dedans l'ame un don de Poësie,

Que Dieu n'a concedé qu'à l'esprit agité

Des poignans aiguillons de sa Divinité,

Quand l'homme en est touché, il devient un Prophete....

Non seulement de plus assiste-t-il à la danse des Muses, mais il a droit à l'initiation particulière d'Euterpe :

Car la gentille Euterpe, ayant ma dextre prise,

Pour m'oster le mortel, par neuf fois me lava

De l'eau d'une fontaine où peu de monde va,

Me charma par neuf fois, puis, d'une bouche enflée

Ayant dessus mon chef son haleine soufflée,

Me herissa le poil de crainte et de fureur

Et me remplist le coeur d'ingenieuse erreur. ...

"Ingénieuse erreur," c'est-à-dire un "je" non seulement animé d'une fureur (erreur), mais aussi rompu aux techniques de l'art (ingénieuse). Et la référence à Dorat et à ces années d'apprentissage vient confirmer cette interprétation. Cette référence peut sembler matérialiser quelque peu le "je," mais cette matérialisation ne dure que l'espace d'un vers, puisque ce "je" ne fait que reprendre les lieux communs de la rhétorique sur la haute poésie.

Disciple de Dorat, qui long temps fut mon maistre,

M'apprist la Poësie, et me monstra comment

On doit feindre et cacher les fables proprement, 
Et à bien desguiser la verité des choses

D'un fabuleux manteau, dont elles sont encloses.

"Desguiser la vérité des choses" pour s'éloigner le plus possible de l'histoire au sens où on l'a définie au début de cet article"... sans user d'aucune fiction, représenter le vray de chacune action."

Car le poète est un prophète "Qui connait la nature et les secrets des Cieux." C'est toujours le "je" de l'Hymne de l'automne qui parle.

Retrouver alors dans les Hymnes à côté de l'énoncé héroïque et de l'énoncé enconomiastique, un énoncé physico-philosophique n'est guère étonnant. La matière des Hymnes de Ronsard est donc de trois sortes : sont chantées les gestes des dieux et des héros, sont loués de grands personnages, sont développées souvent, sous le manteau du mythe, des réflexions philosophiques. Comme on peut le constater, le "je" de l'énoncé rhétorique qui se situe en général au début de l'Hymne cède totalement sa place à un "non-je." ${ }^{10}$ Et l'on pourrait croire qu'avec de tels sujets, la tentation de l'histoire, du vrai, du réel n'était pas imminente.

Il est certain que dans les Hymnes du genre de celle de Castor \& Pollux et de Calays et Zéthès, le débrayage par rapport au référentiel est pratiquement total. Y sont chantés des gestes connues et ce type de récit, pour employer une expression de Louise Milot, sur un tout autre sujet, dans la dernière parution de la Revue Etudes littéraires sur l'énonciation, donne "l'impression de se raconter tout seul." "Dans les hymnes enconomiastiques d'Henry II et de Charles de Lorraine, par contre, et même si la rhétorique avait très bien défini les règles de ce genre, le maintenant et l'ici peuvent donner l'impression de surgir et de faire réapparaitre le réel, l'histoire, dont le grand genre doit s'écarter résolument. Daniel Ménager a bien démontré par quels mécanismes “Ronsard" dans l'Hymne d'Henry II, a su éviter les pièges du rendez-vous avec l'histoire.

L" "Hymne d'Henri II", parce qu'il est construit sur le canevas d'une "institution" princière, tend toujours à dépouiller le roi de sa figure individuelle et historique. Malgré les réferences à l'histoire, les noms propres eux-mêmes interviennent dans ce texte d'une manière ambiguë. Les poètes de la Pléiade, fleurons du règne, ont besoin d'un article pour les présenter :

... un Bellay, un Jodelle,

Un Baif, Pelletier, un Belleau et Tiard.

Ils semblent pris ainsi dans la structure de l'exemple et chargés d'illustrer l'idée de la présence nécessaire de la poésie auprès du roi. Tout se passe d'ailleurs dans ce poème, comme si l'énoncé d'un nom propre restait toujours plus ou moins scandaleux : les grands personnages de la cour d'Henri II n'ont le droit d'être cités que par l'intermédiaire d'une comparaison qui les assimile aux dieux de l'Olympe: Mars pour les guerriers, Mercure pour les diplomates, Neptune pour les amiraux, etc. A sa manière, différente dans sa forme de l'allégorie mais identique à celle-ci pour le résultat, l'“Hymne d'Henri II" 


\section{4 / Renaissance and Reformation}

fausse compagnie à l'histoire ; ou, pour être plus précis, il ne la juge pas assez noble pour pouvoir se passer de la noblesse des dieux. ${ }^{12}$

Dans les hymnes des saisons, le poète cache sous la fiction mythologique le cycle des saisons ou encore leurs attributs particuliers. L'automne devient ainsi une déesse, fille de Nature et du Soleil, fait une alliance avec le vent Auton qui lui servira d'adjuvant, vole à son frère printemps, bouquets et fleurs, à son frère été deux rayons de ses "flammes ardantes" avant de rencontrer Bacchus qui deviendra sur l'heure son époux. Voilà la réalité cachée sous mille symboles.

Comme la matière du Songe de du Bellay qui a trompé bien des critiques, Mme Joukovski écrivait que le Songe "fait appel à un vocabulaire très technique, mais [qu'] il présente un décor imaginaire." Demerson, de son côté affirme : "L'imagination de J. du Bellay est paresseuse : prompte à construire des formes et des couleurs sur le mode de l'hallucination, elle reste passive en face du spectacle, réel mais un peu décevant, des vestiges du passé qui ne la stimulent pas." Et pourtant Gadoffre a magnifiquement démystifié, me semble-t-il, le mystère qui nimbe le Songe pour montrer que cette oeuvre, malgré le nécessaire apparât dont elle devait se revêtir pour prétendre au genre élevé, prend "une allure de pamphlet gallican, ni plus ni moins que les Regrets. Bilan des occasions perdues, des promesses récusées, des usurpations séculières, il a pour arrière-plan les métamorphoses du démon sanglant de Rome qui hante les lieux, mobilise les morts, fascine les vivants. On conçoit que le collaborateur et cousin du cardinal doyen du Sacré Collège ait eu recours aux écrans de fumée. Il ne faisait que dire en code ce que les évêques et archevêques gallicans proclamaient aux Etats généraux, mais il lui était difficile, avec le nom qu'il portait, de formuler en clair une remise en question qui allait beaucoup plus loin et plus au fond des choses que le 'ris sardonien' des Regrets."13

Ceux-ci, par contre, doivent donner l'illusion d'un ré-embrayage à la position originelle du locuteur. Le "je" de l'énoncé rhétorique l'annonçait et en faisait même, on le sait, son programme d'écriture. Finie la fiction, on chante le vrai. Le vrai je malheureux d'ici et de maintenant:

Moy, qui suis malheureux, je plaindray mon malheur

(Sonnet 5)

Maintenant. ..

... mon coeur

Est serf de mille maulx et regrets qui m'ennuyent

(Sonnet 6)

La plainte que je fais (Dilliers) est véritable

(Sonnet 6.77)

La vraie Rome, telle que la voit le "je" malheureux : 
Je ne descouvre icy les mysteres sacrez

Des saincts prestres Romains, je ne veulx rien escrire

Que la vierge honteuse ait vergongne de lire,

Je veulx toucher sans plus aux vices moins secretz.

Il est vrai que l'on pourrait citer ici et là des passages qui semblent d'une vérité déroutante sur l'état de "du Bellay" et sur Rome, sans compter que le fait d'adresser chacun des sonnets ou presque à un locutaire bien identifié crée un effet de réel. Certains s'y sont laissés prendre et émus ont crié à la sincérité, à l'originalité. ${ }^{14}$ Et le "je" de l'énoncé se confondit alors, pour eux, avec le "je" de l'énonciation.

A la lecture d'ensemble, les Regrets apparaissent comme un document vivant, l'exemple d'un journal intime rédigé dans le double mouvement d'un repliement sur soi et d'une observation amusée de la vie extérieure, avec la tendance à dégager de cette expérience une sorte de leçon valable pour le poète et aussi pour les autres, comme un Essai de Montaigne. (Henri Weber)

Il rend un compte impitoyable et de l'état de son coeur qu'il plaint, et de l'état des moeurs, qu'il examine et réprouve. (Albert-Marie Schmidt)

Le principe même de la poésie devient l'expression du sentiment personnel. (Frédéric Boyer)

Et pourtant. . . pourtant Gadoffre rappelle avec raison que "dans la typologie humaniste, la mélancolie est partie intégrante du statut de l'intellectuel, et plus particulièrement du poète." Et il ajoute que Marsile Ficin propose sur ce phénomène "une explication dans la perspective médicale de son temps dans le de Triplici vita : les intellectuels sont victimes d'une consommation excessive de spiritus, l'esprit subtil, émanation du sang qui sert d'instrument à l'âme immatérielle, et cette combustion qui rend le sang plus dense, plus lourd, plus noir, entraîne une dyscrasie mélancolique extrêmement pernicieuse, et surtout néfaste à ceux qui y sont prédestinés par l'astre qui aprésidé à leur naissance : Saturne." 15 Sans compter que le "je" mélancolique de du Bellay, j'ai eu l'occasion de le signaler ailleurs, ${ }^{16}$ reprend les lieux communs de la poésie pétrarquiste aussi bien sur le plan de la technique du sonnet que sur le plan de l'utilisation des images.

Floyd Gray démontrera aussi que malgré le "loyre Gaulois," le "petit lyré," la "douceur Angevine," l'opposition Ulysse/je du sonnet 31 "Heureux qui, comme Ulysse, a fait un beau voyage" est avant tout rhétorique, Ulysse étant le héros épique et le "je" des Regrets, un antihéros empêtré dans son quotidien. "Tout le sonnet, écrit Gray, jouera sur l'opposition poétiquement génératrice du mythe et du quotidien, ce qui correspond à l'équation antithétique Homère/du Bellay, donc deux poétiques contraires, l'une plongeant dans le mythe et l'autre tendant à le détruire."17 
Gadoffre nous apprend même, s'il le fallait, que le personnage du Bellay et le "je" des Regrets n'ont rien en commun :

\begin{abstract}
... la personne qui dit je, l'exilé chagrin, las d'errer, sarcastique, dont les nostalgies s'accordent si bien aux stéréotypes du Romantisme, n'est pas davantage le personnel réel qu'on a voulu y voir. Au témoignage de ceux qui l'ont connu, le du Bellay historique, administrateur perspicace et ferme, est un tout autre homme que l'exilé gémissant des Regrets dans lequel il faut voir-au même titre que dans le mage vaticinant des Antiquitez et du Songe - une "persona," un masque, une création poétique dont le rôle est de médiatiser les sensations et d'orienter un discours. Les malentendus sur Joachim proviennent de cette confusion entre la personne historique et le moi mythique du poète. ${ }^{18}$
\end{abstract}

Quant à la "Rome" des Regrets, elle est, elle aussi, un "acteur" de l'énoncé et il serait bien vain de vouloir y retrouver une Rome historique. Bien sûr, certaines descriptions physiques et humaines prennent l'allure de véritables portraits, au sens rhétorique que donnait à cette expression du Bellay lui-même dans les Regrets. Toutefois, l'on sait, depuis le livre de Gladys Dickinson, l'importance qu'ont eue sur la description des moeurs de Rome les pasquinades, ces satires publiques que l'anonymat populaire affichait sur la fameuse statue de Pasquin. Du Bellay leur doit beaucoup, ainsi qu'à Berni et à bien d'autres. ...

Il serait d'ailleurs fort utile d'étudier attentivement les lieux communs de l'époque sur la Rome des papes, surtout dans le milieu gallican, et de les comparer à ce que l'on retrouve dans les Regrets. L'on constaterait sans doute que "l'auteur" ne va guère plus loin et que la vraie grande réflexion sur Rome se situera dans les Antiquitez et le Songe. Mais ces oeuvres étaient présentées comme étant de haute venue, alors que les Regrets se devaient de créer l'illusion du réel.

Le "je" malheureux, le "maintenant" par rapport à l'autrefois, "l'icy" (Rome) par rapport à la France sont donc les simulacres dont l" auteur" se servira pour satisfaire les exigences rhétoriques du genre.

* * * *

Comparer les Hymnes et les Regrets peut sembler une tâche facile à cause de l'énorme distance entre les deux oeuvres sur le plan de la pratique du texte.

Si l'on comparait par contre les Amours de Cassandre aux Amours de Marie, l'on constaterait que dans cette dernière oeuvre, le "je" de l'énoncé reste toujours le même, alors que le "je" dans Cassandre est constamment projeté vers l'extérieur. Il est tour à tour le "Corèbe insensé" de l'Enéide (s. IV), le Phébus amoureux de Cassandre (s.XXXVI); Prométhée, Ixion; il voudrait être Jupiter, Narcisse. Le "je" n'est plus "je" mais un amoureux mythique d'un mythe non moins tenace, Cassandre. $\mathrm{La}$ 
Cassandre de Troyes, prophétesse, inflexible, Nymphe, guerrière, beauté divine. .. Cassandre, maitresse fabuleuse, mythique, dont la dénomination même est génératrice de sèmes féconds, propres à assurer au poème l'épaisseur nécessaire a l'élévation poétique. Les exemples pourraient être nombreux : je me contenterai de citer un sonnet parmi les moins couramment évoqués, mais dont la valeur exemplaire est remarquable :

Apres ton cours je ne haste mes pas

Pour te souiller d'une amour deshonneste :

Demeure donq : Le Locroys m'amonneste

Aux bordz Gyrez de ne te forcer pas.

Neptune oyant ses blasphemes d'abas,

Accabla là son impudique teste

D'un grand rocher au fort de la tempeste.

Le ciel conduit le meschant au trespas.

Il te voulut, le meschant, violer,

Lors que la peur te faisoit accoller

Les piedz vangeurs de sa Grecque Minerve :

Moy je ne veulx qu'à ta grandeur offrir

Ce chaste cuoeur, s'il te plaist de souffrir

Qu'en l'immolant de victime il te serve. ${ }^{19}$

Marie par contre est de Bourgueil, une fille d'Anjou, celle que l'on affuble de diminutifs, de qualificatifs non mythiques (Mon doux printemps, Ma douce fleur nouvelle, Mon doux plair, Ma douce colombelle. . .). Bien sûr, Marie est aussi une création du poète, un acteur de l'énoncé, mais alors que dans les Amours de Cassandre tout concourait à sortir le "je" de Cassandre de l'illusion référentielle, dans les Amours de Marie, les simulacres sont tenaces pour créer l'illusion du vrai. Car la "mutation poétique profonde" qui s'opère du livre des amours à celui de la Continuation, pour reprendre l'expression de Rigolot, ${ }^{20}$ les "couleurs opposées" qui marquent ces deux oeuvres sont le résultat d'un choix littéraire évident et dont rendent compte les énoncés rhétoriques suivants, puis̀s dans chacune des oeuvres :

Divin troupeau, qui sur les rives molles

Du fleuve Eurote, ou sur le mont natal,

Ou sur le bord du chevalin crystal,

Assis, tenez vos plus sainctes escolles :

Si quelque foys aux saultz de vos carolles

M'avez receu par ung astre fatal,

Plus dur qu'en fer, qu'en cuyvre ou qu'en metal,

Dans vostre temple engravez ces paroles :

RONSARD, AFFIN QUE LE SIECLE A VENIR,

DE PERE EN FILZ SE PUISSE SOUVENIR,

D'UNE BEAUTE QUI SAGEMENT AFFOLE, 
DE LA MAIN DEXTRE APPEND A NOSTRE AUTEL, L'HUMBLE DISCOURS DE SON LIVRE IMMORTEL, SON CUOEUR DE L'AUTRE, AUX PIEDZ DE CESTE IDOLE. ${ }^{21}$

Dy luy que les amours ne se souspirent pas

D'un vers hautement grave, ains d'un beau stille bas, Populaire \& plaisant, ainsi qu'a fait Tibulle, L'ingenieux Ovide, \& le docte Catulle :

Le fils de Venus hait ces ostentations :

Il sufist qu'on luy chante au vray ses passions,

Sans enfleure ny fard, d'un mignard $\&$ dous stille,

Coulant d'un petit bruit comme une eau qui distille. ${ }^{22}$

La poésie élevée doit donner l'impression qu'elle s'éloigne du réel, le plus qu'il est possible : il s'agit alors d'opaciser les écrans et le recours au mythe en est le motif privilégié, situant l'acte d'énonciation, le plus parfaitement possible, en dehors de l'ego, du hic et du nunc du sujet d'énonciation. La poésie humble par contre doit donner l'apparente illusion de dire vrai sans fiction aucune : elle recourra donc aux simulacres d'unje, d'un ici et d'un maintenant pour créer l'impression que l'acte d'énonciation se confond avec le sujet de l'énonciation et ainsi avec le réel et la vérité. Les oeuvres nous le démontrent mais aussi la réflexion rhétorique qui commence ces oeuvres. Dire le comment (les préfaces, les pièces liminaires) est donc aussi important que de dire le quoi. N'est-ce pas la mise en pratique de la réconciliation Nature - Art? Les poètes sont des inspirés, bien sûr, mais ils connaissent aussi leur métier. Les Muses ne veulent pas que l'on force leurs portes, bien évidemment, mais elles veulent un poète artificieux.

Bien te veux-je avertir de chercher la solitude \& le silence amy des Muses, qui aussi (affin que ne laisses passer cette fureur divine, qui quelquesfois agite \& echaufe les esprits poëtiques, \& sans la quele ne fault point que nul espere faire chose qui dure) n'ouvrent jamais la porte de leur sacré cabinet, si non à ceux qui hurtent rudement. ${ }^{23}$

Tout n'est pas grande poésie, haute inspiration, mais tout doit se faire avec art, avec métier, puisque c'est là justement que réside l'originalité.

Université Laval 


\section{Notes}

1 Boulanger, André. L'art poëtique de Jacques Pelletier du Mans. (Paris : Les Belles Lettres, 1930), p. 176 et ss.

2 Ibid., p. 179.

3 Ibid., p. 194.

4 Ronsard, Oeuvres complètes, t. VIII, Les Hymnes de 1555, le second livre des Hymnes de 1556, éd. critique de Paul Laumonier (Paris : Didier, 1966), p. XII.

5 Du Bellay, Joachim, Les Regrets et autres oeuvres poëtiques suivis des Antiquitez de Rome plus un Songe ou une Vision sur le mesme subject, texte établi par J. Jolliffe, introduit et commenté par M.A. Screech (Genève : Droz, T.L.F., 1960), s. 4, p. 59.

6 Ces oppositions sont tirées de mon article "Théorie et pratique dans les Regrets de du Bellay," Ren. \& Réf., 13 (1977), 47 et s.

7 Du Bellay, Joachim, Oeuvres poétiques, t. VI, Discours et traductions, éd. critique publiée par Henri Chamard, (Paris : Droz, S.T.F.M., 1931), p. 164 et s.

8 Ronsard, Oeuvres complètes, éd. Laumonier, t. XVI, p. 4.

9 Sémiotique. Dictionnaire raisonné de la théorie du langage (Hachette : Université, 1979), article "débrayage," par. B, p. 79.

10 Le "je" qui réapparaît à la fin de chacun des Hymnes pratiquement pour louer et supplier ne fait que suivre les règles propres à un tel genre et il n'en répète que les lieux communs.

11 Etudes littéraires, 16 (avril, 1983) 138.

12 Menager, Daniel. Ronsard, le roi, le poète et les hommes (Genève : Droz, 1979), p. 43.

13 Du Bellay et le sacré (Paris : Gallimard, 1978), p. 181 et s. Les citations de Joukovski et de Demerson sont tirées de cet ouvrage respectivement aux pages 156 et 157.

14 Bellenger, Yvonne, Du Bellay: ses "Regrets" qu'il fit dans Rome ... (Paris : Nizet, 1975). Ces citations sont prises au chapitre II parmi divers jugements sur les Regrets.

15 Opus cit., p. 11 et s.

16 Article paru dans Renaissance et Réforme et déjà cité.

17 Gray, Floyd. La poétique de du Bellay (Paris : Nizet, 1978).

18 Opus cit., p. 127 ts. p. 117.

19 Oeuvres complètes, t. IV les Amours, Ed. Laumonier, s. 79, p. 80.

20 Rigolot, François, Poétique et onomastique (Genève : Droz, 1977), p. 210.

21 Ronsard, Oeuvres complètes, t. IV Les Amours (1552), èd. Laumonier (1957), p. 4.

22 Ronsard, Oeuvres complètes, $t$.VI, Les Odes de 1555, Les continuations des Amours 15551556, éd. Laumonier (1959), p. 324.

23 Du Bellay, Joachim, La deffence et illustration de la langue francoyse, éd. crit. publiée par $\mathrm{H}$. Chamard, 2e tirage (Paris : Didier, 1961), p. 169 ets. 